\title{
Cumulative Hazard Intensity Based on Sequential Preventive Maintenance Strategy for Ensuring Minimum Reliability
}

\author{
Juhyun Lee ${ }^{\# 1}$, Jihyun Park ${ }^{\# 2}$, Suneung Ahn ${ }^{* 3}$ \\ \# Department of Industrial and Management Engineering, Hanyang University \\ 222, Wangsimni-ro, Seongdong-gu, Seoul, 04763, Korea \\ ${ }^{1}$ ljh812@ hanyang.ac.kr \\ 2 pjh3226@hanyang.ac.kr \\ * Department of Industrial and Management Engineering, Hanyang University ERICA Campus \\ 55 Hanyangdeahak-ro, Sangnok-gu, Ansan, Gyeonggi-do, 15588, Korea \\ ${ }^{3}$ sunahn@hanyang.ac.kr
}

\begin{abstract}
In designing a preventive maintenance policy, considering only either system reliability or cost may not ensure sustainable system operation because the biased design of a PM policy can result in high maintenance cost and short system life. A PM policy should thus be designed considering a trade-off between system reliability and maintenance costs. PM actions in condition-based maintenance policies are commonly performed at a fixed reliability threshold, which is set high to prevent severe breakdowns. However, it may be difficult to establish cost-effectiveness of a model when the reliability threshold is constrained to be fixed. In this study, a cost-effective PM policy based on a reliability threshold that decreases as the frequency of PM actions increases, is developed, which considers a fixed cumulative hazard threshold while keeping the reliability above an acceptable level. The developed policy was analyzed in two cases; one where the cumulative hazard threshold and one where the predetermined minimum reliability were given as the condition variable, respectively. The results were compared to the model of the fixed reliability threshold, which showed that the proposed PM model led to a lower expected maintenance cost and longer system lifespan. Finally, sensitivity analyses of the condition variables and the maintenance costs were conducted.
\end{abstract}

Keywords: Maintenance, Reliability threshold, Minimal repair, Cumulative hazard threshold

\section{INTRODUCTION}

Preventive maintenance (PM) policies are considered to be a critical part in engineering fields. In practice, PM actions for engineering systems, such as car, airplane, and ships, devote to reducing breakdowns caused by wear-out and to extend system lifespan. Moreover, in designing PM policies, it should consider a trade-off between reliability and cost. PM policies biased in terms of reliability may result in high maintenance cost and poor system availability caused by frequent PM actions. In contrast, insufficient PM actions make system lifespan shortened.

Ever since Barlow and Hunter [1] proposed a preventive replacement policy with minimal repairs, many researches on $\mathrm{PM}$ policies have been conducted to deal with the trade-off problem. Toshio Nakagawa [2] proposed an imperfect PM policy. Canfield [3] proposed the slow degradation process. Yeh and Chang [4] proposed a sequential PM policy with a failure rate threshold, and applied it to leased equipment. Zhao [5] proposed a PM policy considering the critical reliability level in a degraded state. Zhou, Xi, and Lee [6] proposed a reliabilitycentered predictive maintenance policy using reliability threshold.

PM actions of traditional works are carried out despite the sufficiently good state of the system; this is attributed to the constraint of the reliability threshold, resulting in inefficient PM actions. The inefficient PM actions are frequently carried out because of the decreasing PM intervals, which lead to a high maintenance cost. It may be difficult to extend system lifespan because of high maintenance cost. As a result, cost-effectiveness may be difficult to be established via the PM model when the reliability threshold is fixed. It may also be difficult to prevent frequent PM actions due to the constraint that the reliability threshold is set high.

The main objective of PM actions is not only to prevent severe breakdowns, but also to ensure sustainable system operation. In the present study, a model was developed to ensure sustainable system operation. The proposed model uses the cumulative hazard intensity as the condition variable, instead of using the reliability. The cumulative hazard threshold can be used to calculate the unfixed reliability 
Juhyun Lee et al., International Journal of Computing, Communications and Networking, 7(2) April - June 2018, 1-7

threshold that lowers as the frequency of PM actions increases. The unfixed reliability threshold can prevent inefficient PM actions owing to longer PM intervals than those of model with a fixed reliability threshold, while ensuring a certain system reliability to be preferred by field managers. Therefore, this study proposes a cost-effective PM policy based on the cumulative hazard threshold, which aims to reduce maintenance cost and to extend system lifespan.

This paper is organized as follows. Section 2 explains notation and assumptions to establish the proposed PM model, and derives the expected maintenance cost function. In addition, the reliability constraints based on the cumulative hazard threshold are derived. Section 3 defines two cases and provides an algorithm to find the optimal solution. Section 4 provides a numerical example to illustrate the proposed PM model, and compares the result with that obtained from the model of a fixed reliability threshold. Sensitivity analyses are also conducted to investigate critical elements. Finally, Section 5 discusses the conclusions of this research.

\section{Preventive maintenance POLICy BaSed ON CUMULATIVE HAZARD INTENSITY}

\section{A. Notation and Assumption}

\section{Notation}

$\alpha \quad$ scale parameter of hazard intensity

functions, where $\alpha>0$

$\beta \quad$ deterioration parameter of hazard intensity functions, where $\beta>1$

$\rho_{i} \quad$ age reduction factor, where $0<\rho_{1}<\rho_{2}<\cdots<\rho_{N-1}<1$, $i=1,2, . ., N-1$

$R(t)$ reliability functions without PM at time $t$

$m_{i} \quad$ PM interval at the $i$ th PM action, where $i=1,2, . ., N$

$T_{i} \quad$ effective age of the $i$ th PM action, where $T_{i}=\sum_{j=1}^{i} m_{j}, i=1,2, . ., N$

$T_{o} \quad$ system lifetime until replacement, $T_{o}=\sum_{j=1}^{N} m_{j}$

$M_{r} \quad$ number of minimal repair actions until system replacement

$c_{r} \quad$ cost for minimal repair action

$c_{m} \quad$ cost for preventive maintenance action

$c_{i} \quad$ cost for replacement of system

$$
\begin{array}{ll}
n_{c} & \text { cumulative hazard threshold } \\
R_{1} & \text { critical reliability level } \\
R_{1, i} & \text { system reliability at } i \text { th PM action } \\
R_{G} & \text { guaranteed minimum reliability in case } 1 \\
\tilde{R} & \text { predetermined minimum reliability in case }
\end{array}
$$

The assumptions are summarized in detail, as follows:

\section{Assumptions}

1. When the cumulative hazard intensity reaches the predetermined threshold $n_{c}, \mathrm{PM}$ action is immediately performed.

2. The non-homogeneous Poisson process (NHPP) is considered to be a deterioration process. It can be modeled by the Weibull power-law failure intensity given as $h(t)=\alpha \beta t^{\beta-1}$.

3. When system fails, the minimal repair is immediately performed, and then, the system is restored to the previous state. In addition, the durations related to maintenance action are negligible.

4. Age reduction factor, $\rho_{i}$, is used to represent imperfect PM action, where $0<\rho_{1}<\rho_{2}<\cdots<\rho_{N-1}<1$.

5. The system is replaced at the $N$ th PM action.

6. The PM cost $c_{m}$, the minimal repair cost $c_{r}$, and the replacement cost $c_{i}$, are regarded to be constant.

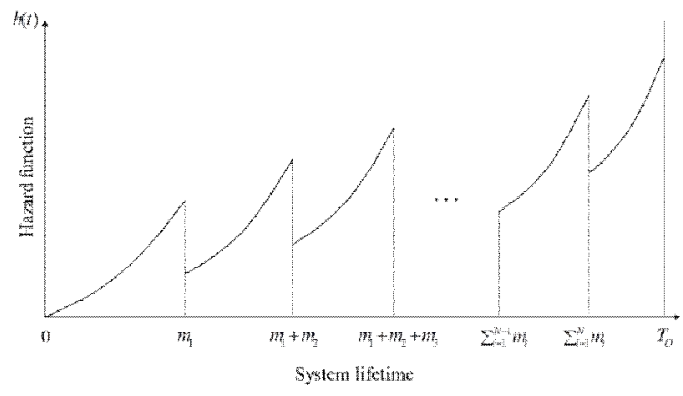

Fig 1. Hazard intensity functions under the proposed PM model 
Juhyun Lee et al., International Journal of Computing, Communications and Networking, 7(2) April - June 2018, 1-7

\section{B. Preventive maintenance model based on the cumulative hazard threshold}

In the present study, imperfect PM actions are modeled as a PAS model [7]. The PAS model is appropriate to represent the efficiency of imperfect PM actions. The age reduction factor in PM policies has been widely used in related research ([8], [9], [10]). The system effective age after the $(i-1)$ th PM action can be restored from $T_{i-1}$ to $\rho_{i-1} T_{i-1}$. The system effective age at the $i$ th PM action can be determined as

$$
\begin{aligned}
T_{i} & =m_{i}+\rho_{i-1} T_{i-1} \\
& =m_{i}+\rho_{i-1}\left(m_{i-1}+\rho_{i-2} T_{i-2}\right) \\
& =m_{i}+\rho_{i-1} m_{i-1}+\cdots+\left(\prod_{j=1}^{i-1} \rho_{j}\right) T_{1},
\end{aligned}
$$

for $i=1,2, \ldots, N$. The $i$ th $\mathrm{PM}$ interval can be calculated as

$$
m_{i}=T_{i}-\rho_{i-1} T_{i-1},
$$

for $i=1,2, \ldots, N$. The system lifespan can be calculated as

$$
\begin{aligned}
T_{o} & =\sum_{i=1}^{N} m_{i} \\
& =T_{N}+\sum_{i=1}^{N-1}\left(1-\rho_{i}\right) T_{i} .
\end{aligned}
$$

The cumulative hazard intensity, $H(t)$ represents the expected number of breakdowns up to time $t$ according to the definition of NHPP. The cumulative hazard threshold indicates the expected number of system breakdowns in each PM cycle, and can be calculated to a specific ratio indicating a critical reliability level by using the relationship of between reliability and cumulative hazard intensity. Hence, every PM action in this study was performed when either the critical reliability level or the number of system breakdowns reaches a predetermined value, which is expressed as

$$
\begin{array}{r}
\exp \left(-\int_{0}^{T_{1}} h(t) d t\right)=\exp \left(-\int_{\rho_{1} T_{1}}^{T_{2}} h(t) d t\right) \\
=\cdots=\exp \left(-n_{c}\right)=R_{1},
\end{array}
$$

which is expressed through a critical reliability level $R_{1}$ for $0 \leq R_{1} \leq 1$. Based on the logarithm of Eq. 4, the relationship between the cumulative hazard threshold and the critical reliability level can be derived as

$$
\int_{0}^{T_{1}} h(t) d t=\int_{\rho_{1} T_{1}}^{T_{2}} h(t) d t=\cdots=n_{c}=-\ln R_{1} .
$$

The number of minimal repair actions during system lifespan can be determined as:

$$
\begin{aligned}
M_{r} & =\int_{0}^{T_{1}} h(t) d t+\cdots+\int_{\rho_{N-1} T_{N-1}}^{T_{N}} h(t) d t \\
& =n_{c}+\cdots+n_{c} \\
& =N n_{c}=N\left(-\ln R_{1}\right) .
\end{aligned}
$$

The maintenance cost per unit time up to system replacement can be derived as

$$
C(N)=\frac{n_{c} N c_{r}+(N-1) c_{m}+c_{i}}{T_{o}} .
$$

\section{Derivation of reliability constraints based on cumulative hazard threshold}

The reliability function of the initial system is given as

$$
R(t)=\exp \left(-\int_{0}^{t} h(u) d u\right)
$$

where $R(t)$ denotes system reliability at time $t$ where $0 \leq R(t) \leq 1$ and $H(t)$ denotes the cumulative hazard intensity at time $t$. It can be observed that the reliability and the cumulative hazard intensity are in inverse proportion to each other. Eq. 4 can be rewritten as

$$
\begin{aligned}
\exp \left(-H\left(T_{1}\right)\right) & =\exp \left(-H\left(T_{2}\right)+H\left(\rho_{1} T_{1}\right)\right) \\
& =\cdots=R_{1} .
\end{aligned}
$$

Eq. 9 shows that a critical reliability level $R_{1}$ in every PM cycle is equal to a specific ratio. The critical reliability level does not indicate a reliability threshold, but an acceptable level of system breakdowns in each PM cycle ([5]). The acceptable level of system breakdowns (more specifically, the cumulative hazard threshold) can be used to calculate an unfixed reliability threshold on the model. In the proposed model, the number of system breakdowns remains the same in every PM cycle because of the constraint that the cumulative hazard threshold is fixed. The reliability constraints of this study are given as

$$
\begin{aligned}
\exp \left(-H\left(T_{i}\right)\right) & =R_{1} \exp \left(-H\left(\rho_{i-1} T_{i-1}\right)\right) \\
& =R_{1, i},
\end{aligned}
$$

where $i=1,2, \ldots, N$, where $R_{1, i}$ denotes the reliability threshold at the $i$ th PM action as obtained by solving Eq. 9 with respect to the cumulative hazard intensity at the effective age of the $i$ th PM. where $i=1,2, \ldots, N$ and $R_{k}$ indicates the fixed reliability threshold. The unfixed reliability threshold 
in the proposed model decreases as the frequency of PM actions increases, while the system reliability is maintained above an acceptable level. For example, if the first PM action is performed at system reliability $R_{1}$, the system reliability is then restored by an age reduction factor from $R_{1}$ to $R_{2}$. The second PM action is performed at a system reliability of $R_{1} R_{2}$. By using Eq.10, the unfixed reliability threshold at the $i$ th PM action can be derived as

$$
\begin{aligned}
R_{1, i} & =R_{1} R_{i} \\
& =R_{1}^{S(i)},
\end{aligned}
$$

where $S(i)=1+\sum_{z=1}^{i-1} \prod_{j=z}^{i-1} \rho_{j}^{\beta} \quad$ in that case of $0<\rho_{1}<\rho_{2}<\cdots<\rho_{N-1}<1$. When the frequency of $\mathrm{PM}$ actions increases to reach an infinitely high value, the power function of Eq. 12, converges to a specific value. The specific value is used in the calculation of the guaranteed minimum reliability. The guaranteed minimum reliability means that system reliability is guaranteed by the proposed PM policy.

\section{DETERMINATION OF THE OPTIMAL PM POLICY}

Here, we try to design an optimal sequential PM policy considering two cases that can help illustrating a maintenance cost.

Case 1: Every PM action is immediately performed when the cumulative hazard intensity reaches a predetermined threshold. The cumulative hazard threshold is set low enough to prevent inefficient PM actions from being implemented to the system, and it is used to calculate the guaranteed minimum reliability. Note that the decision variable is the frequency of PM actions, $(N-1)$, until system replacement.

Case 2: A predetermined minimum reliability is provided to the field manager. It is set high enough to prevent severe breakdowns, and it is used to calculate the cumulative hazard threshold. Every PM action is performed when the cumulative hazard intensity reaches a calculated threshold. It should be noted that the predetermined minimum reliability can be higher than the guaranteed minimum reliability in case 1 .

\section{A. Algorithm for the optimal PM policy}

Using the assumptions stated in section 2, the cost function can be re-derived as

$$
C(N)=\frac{-\ln R_{1} N c_{r}+(N-1) c_{m}+c_{i}}{T_{N}+\sum_{i=1}^{N-1}\left(1-\rho_{i}\right) T_{i}}
$$

The guaranteed minimum reliability $R_{G}$ considered in case 1 can be calculated via the age reduction factor and the cumulative hazard threshold, and can be given as

$$
R_{G}=R_{1}^{\lim _{i \rightarrow \infty} S(i)}
$$

Solving the constraint in Eq. 4 with respect to $T_{i}$, and substituting $T_{i}$ into Eq. 2, the time interval between the $(i-1)$ th and the $i$ th PM actions can be calculated as

$$
m_{i}=\left\{\left(1+\sum_{z=1}^{i-1} \prod_{j=z}^{i-1} \rho_{j}^{\beta}\right)^{1 / \beta}-\rho_{i-1}\left(1+\sum_{z=1}^{i-2} \prod_{j=z}^{i-2} \rho_{j}^{\beta}\right)^{1 / \beta}\right\} m_{1},
$$

where $i=1,2, . ., N$. The reliability threshold at the $i$ th $\mathrm{PM}$ action can be re-derived as

$$
R_{1, i}=R_{1}^{S(i)}
$$

where $i=1,2, . ., N$. The predetermined minimum reliability $\tilde{R}$ considered in case 2 can be used to calculate the fixed cumulative hazard threshold and can be obtained as

$$
n_{c}=-\ln \tilde{R}^{\frac{1}{\lim _{i \rightarrow \infty} S(i)}}
$$

Additionally, a necessary condition to obtain the optimal solution $N^{*}$ can be expressed by Eq. 17 .

$$
C\left(N^{*}+1\right) \geq C\left(N^{*}\right)<C\left(N^{*}-1\right) .
$$

The optimal solution can be obtained via the following algorithm:

\section{Algorithm}

Step1: If the cumulative hazard threshold $n_{c}$ is provided, then compute the guaranteed minimum reliability which is given in Eq. 13 and proceed to step 3; else, if the predetermined minimum reliability is provided, go to step 2 .

Step 2: Compute the cumulative hazard threshold $n_{c}$, which is given in Eq. 16; then, go to step 3 . 
Juhyun Lee et al., International Journal of Computing, Communications and Networking, 7(2) April - June 2018, 1-7

Step 3: Set $N=1$ and calculate the maintenance cost per unit time $C(N=1)$, which is given in Eq. 12 ; then, go to step 4.

Step 4: Let $N=N+1$. Calculate the maintenance cost per unit time $C(N+1)$, which is given in Eq. 12; then, go to step 5.

Step 5: If $C(N)<C(N+1)$ then attain the optimal solution $N^{*}$, stop the process, and go to step 6; else, go to step 4.

Step 6: Using the optimal solution $N^{*}$, compute the PM intervals $\underline{m}=\left(m_{1}, m_{2}, \ldots, m_{N^{*}}\right)$, which is given in Eq. 14 and the reliability threshold $\underline{R}=\left(R_{1,1}, R_{1,2}, \ldots, R_{1, N^{*}}\right)$, which is given in Eq. 15 , for $i=1,2, \ldots, N^{*}$.

\section{NUMERICAL EXAMPLE}

In order to demonstrate the proposed PM policy, a numerical example is shown and the result is compared with the model of the fixed reliability threshold ([11]). The parameters of the hazard intensity function were assumed to be $\alpha=1.8$ and $\beta=2.6$, and the age reduction factor was given as $\rho_{i}=i /(2 i+1)$ ([8], [9]) which increased as the frequency of PM actions increased. The critical reliability level was given as $R_{1}=0.9$. The cumulative hazard threshold, $n_{c}=-\ln (0.9)$, in case 1 could be calculated via the critical reliability level. The predetermined minimum reliability for solving the case 2 was given as $\tilde{R}=0.9$. The fixed reliability threshold was given the same as the critical reliability level. The related costs for maintenance action were considered as their ratios, which were given as $c_{r} / c_{m}=0.7$ and $c_{i} / c_{m}=2.7$. PM cost was set higher than the minimal repair cost. The result is summarized in Table 1 and Fig. 2.

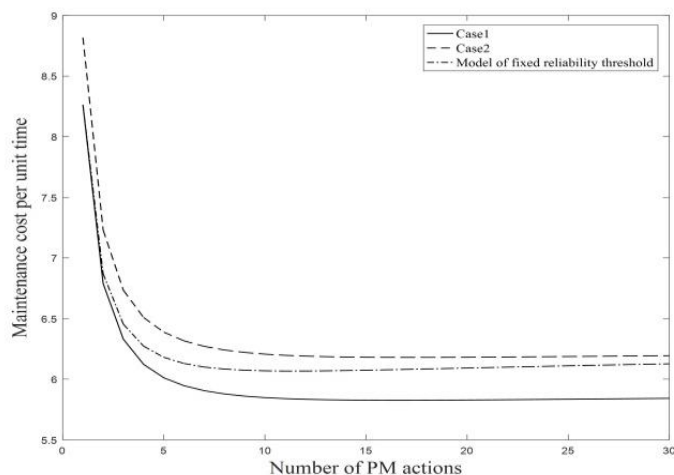

Fig 2. Maintenance cost per unit time as frequency of PM actions increases

From Table 1, the maintenance cost per unit time in case 1 is incurred lower than the optimal ones obtained from both case 2 and the model of fixed reliability threshold. The system lifespan in case 1

TABLE I

Optimal solution and system performance obtained by the proposed algorithm

\begin{tabular}{|c|c|c|c|}
\hline \multirow{2}{*}{ Model } & \multirow{2}{*}{$\begin{array}{l}\text { Model of fixed } \\
\text { reliability threshold }\end{array}$} & \multicolumn{2}{|c|}{ The proposed model } \\
\hline & & Case1 & Case2 \\
\hline System lifespan & 2.2123 & 3.4249 & 2.1587 \\
\hline Minimum reliability & 0.9000 & 0.8815 & 0.9000 \\
\hline Maintenance cost & 6.0670 & 5.8261 & 6.1796 \\
\hline Number of PM actions & 11 & 17 & 11 \\
\hline
\end{tabular}

TABLE II

The PM interval and the reliability threshold of the optimal solution

\begin{tabular}{|c|c|c|c|c|c|c|}
\hline \multirow{3}{*}{$\begin{array}{c}\text { Model } \\
N^{*}\end{array}$} & \multirow{2}{*}{\multicolumn{2}{|c|}{$\begin{array}{c}\text { Model of fixed reliability } \\
\text { threshold }\end{array}$}} & \multicolumn{4}{|c|}{ The proposed model } \\
\hline & & & \multicolumn{2}{|c|}{ Case1 } & \multicolumn{2}{|c|}{ Case2 } \\
\hline & $m_{i}$ & $R_{i, N^{*}}$ & $m_{i}$ & $R_{i, N^{*}}$ & $m_{i}$ & $R_{i, N^{*}}$ \\
\hline 1 & 0.3357 & 0.9000 & 0.3357 & 0.9000 & 0.3132 & 0.9158 \\
\hline 2 & 0.2238 & 0.9000 & 0.2311 & 0.8946 & 0.2156 & 0.9112 \\
\hline 3 & 0.2014 & 0.9000 & 0.2107 & 0.8908 & 0.1966 & 0.9079 \\
\hline 4 & 0.1918 & 0.9000 & 0.2017 & 0.8886 & 0.1882 & 0.9061 \\
\hline 5 & 0.1865 & 0.9000 & 0.1967 & 0.8872 & 0.1835 & 0.9049 \\
\hline 6 & 0.1831 & 0.9000 & 0.1935 & 0.8862 & 0.1806 & 0.9041 \\
\hline 7 & 0.1808 & 0.9000 & 0.1914 & 0.8856 & 0.1785 & 0.9035 \\
\hline 8 & 0.1790 & 0.9000 & 0.1898 & 0.8850 & 0.1771 & 0.9031 \\
\hline 9 & 0.1777 & 0.9000 & 0.1886 & 0.8846 & 0.1760 & 0.9027 \\
\hline 10 & 0.1767 & 0.9000 & 0.1877 & 0.8843 & 0.1751 & 0.9024 \\
\hline 11 & 0.1758 & 0.9000 & 0.1869 & 0.8841 & 0.1744 & 0.9022 \\
\hline 12 & & & 0.1863 & 0.8839 & & \\
\hline 13 & & & 0.1858 & 0.8837 & & \\
\hline 14 & & & 0.18535 & 0.8835 & & \\
\hline 15 & & & 0.1849 & 0.8834 & & \\
\hline 16 & & & 0.1846 & 0.8833 & & \\
\hline
\end{tabular}


Juhyun Lee et al., International Journal of Computing, Communications and Networking, 7(2) April - June 2018, 1-7

TABLE III

Sensitivity analysis for condition variables (given that $c_{r} / c_{m}=0.7, c_{i} / c_{m}=2.7, \alpha=1.8, \beta=2.6$, and $n_{c}=-\ln \left(R_{1}\right)$ )

\begin{tabular}{|c|c|c|c|c|c|c|c|}
\hline \multicolumn{8}{|c|}{ The proposed model } \\
\hline \multirow[b]{2}{*}{$n_{c}\left(R_{1}\right)$} & \multicolumn{3}{|c|}{ Case1 } & \multirow[b]{2}{*}{$\tilde{R}$} & \multicolumn{3}{|c|}{ Case2 } \\
\hline & $N^{*}$ & $T_{o}$ & $C\left(N^{*}\right)$ & & $N^{*}$ & $T_{o}$ & $C\left(N^{*}\right)$ \\
\hline $0.2095(0.8110)$ & 13 & 3.4983 & 4.7470 & 0.8110 & 14 & 3.4892 & 4.9909 \\
\hline $0.1732(0.8410)$ & 14 & 3.4756 & 5.0055 & 0.8410 & 15 & 3.4517 & 5.2781 \\
\hline $0.1393(0.8700)$ & 15 & 3.4021 & 5.3386 & 0.8700 & 16 & 3.3660 & 5.6455 \\
\hline $0.1054(0.9000)$ & 17 & 3.4249 & 5.8261 & 0.9000 & 17 & 3.1955 & 6.1796 \\
\hline $0.0726(0.9300)$ & 18 & 3.1268 & 6.5927 & 0.9300 & 18 & 2.9174 & 7.0143 \\
\hline $0.0419(0.9590)$ & 19 & 2.6594 & 7.9929 & 0.9590 & 20 & 2.6015 & 8.5296 \\
\hline $0.0111(0.9890)$ & 21 & 1.7482 & 13.0780 & 0.9890 & 21 & 1.6311 & 14.0004 \\
\hline
\end{tabular}

TABLE IV

Sensitivity analysis for maintenance costs (given that $c_{r} / c_{m}=0.7, c_{i} / c_{m}=2.7, \alpha=1.8$, and $\beta=2.6$ )

\begin{tabular}{|c|c|c|c|c|c|c|c|c|}
\hline \multirow{2}{*}{$c_{r}$} & \multirow{2}{*}{$c_{i}$} & \multirow{2}{*}{$c_{m}$} & \multicolumn{7}{|c|}{ The proposed model } \\
\cline { 5 - 10 } & & & \multicolumn{3}{|c|}{ Case1 } & \multicolumn{3}{c|}{ Case2 } \\
\cline { 5 - 10 } & & & $N^{*}$ & $T_{o}$ & $C\left(N^{*}\right)$ & $N^{*}$ & $T_{o}$ & $C\left(N^{*}\right)$ \\
\hline 0.56 & 2.70 & 1.00 & 17 & 3.4249 & 5.7529 & 18 & 3.3672 & 6.1139 \\
\hline 0.63 & 2.70 & 1.00 & 17 & 3.4249 & 5.7895 & 18 & 3.3672 & 6.1468 \\
\hline 0.70 & 2.70 & 1.00 & 17 & 3.4249 & 5.8261 & 17 & 3.1955 & 6.1796 \\
\hline 0.77 & 2.70 & 1.00 & 16 & 3.2406 & 5.8626 & 17 & 3.1955 & 6.2124 \\
\hline 0.84 & 2.70 & 1.00 & 16 & 3.2406 & 5.8990 & 17 & 3.1955 & 6.2452 \\
\hline 0.70 & 2.16 & 1.00 & 6 & 1.3694 & 5.5519 & 6 & 1.2776 & 5.8933 \\
\hline 0.70 & 2.43 & 1.00 & 9 & 1.9391 & 5.7211 & 10 & 1.9843 & 6.0706 \\
\hline 0.70 & 2.70 & 1.00 & 17 & 3.4249 & 5.8261 & 17 & 3.1955 & 6.1796 \\
\hline 0.70 & 2.97 & 1.00 & 30 & 5.8048 & 5.8886 & 31 & 5.5861 & 6.2439 \\
\hline 0.70 & 3.24 & 1.00 & 54 & 10.1648 & 5.9246 & 57 & 9.9912 & 6.2806 \\
\hline 0.70 & 2.70 & 0.80 & 67 & 12.5190 & 4.8280 & 72 & 12.5245 & 5.1047 \\
\hline 0.70 & 2.70 & 0.90 & 31 & 5.9871 & 5.3425 & 32 & 5.7561 & 5.6585 \\
\hline 0.70 & 2.70 & 1.00 & 17 & 3.4249 & 5.8261 & 17 & 3.1955 & 6.1796 \\
\hline 0.70 & 2.70 & 1.10 & 10 & 2.1267 & 6.2713 & 10 & 1.9843 & 6.6602 \\
\hline 0.70 & 2.70 & 1.20 & 7 & 1.5607 & 6.6741 & 7 & 1.4562 & 7.0947 \\
\hline
\end{tabular}

was longer than that of both cases because more system breakdowns in case 1 were allowed; hence, the PM intervals in case 1 were longer. In addition, the result in case 2 presents not only a short system lifespan but also a high maintenance cost, as well. However, the system reliability in case 2 was maintained higher than that of both case 1 and the model of fixed reliability threshold because the predetermined minimum reliability indicated that the frequency of PM actions was an infinitely high.

The PM intervals and the reliability threshold are summarized in Table $\square$. As shown in Table $\square$, the PM intervals of both models decreased due to the deterioration process. In this study, the reliability threshold decreased because of the fixed cumulative hazard threshold, unlike the model of the fixed reliability threshold. The PM intervals in case 1 were longer than those of both case 2 and the model of the fixed reliability threshold because of the low reliability threshold in case 1 . In contrast, the PM intervals in case 2 were shorter than those of both case 1 and the model of the fixed reliability threshold.

The sensitivity analyses for related costs on maintenance action were conducted in the following cases: $c_{r}$ increased from 0.56 to $0.84, c_{i}$ increased from 2.16 to 3.24 , and $c_{m}$ increased from 0.80 to 1.20 . The results are summarized in Table $\square$.

As shown in Table $\square, N^{*}$ was influenced by the change in related costs for maintenance actions. As the replacement cost decreased, the system was quickly replaced, as the effect on the replacement cost in an optimal PM policy increased. In contrast, as the PM cost decreased, the system was slowly replaced because the effect on the PM cost in an optimal PM policy decreased. However, the adjustment for the minimal repair cost had less influence on the optimal solution than the adjustment for both the PM cost and the replacement cost. Moreover, the results of the sensitivity analyses for maintenance costs can help the field manager 
determine the costs level to derive an optimal PM policy. Whether the field manager decides the reduction of or the investment in related costs for maintenance actions up to $10 \%$, the reduction of the PM cost or the investment in replacement costs can be a reasonable decision-making outcome.

\section{Conclusion}

Typically, the biased design of PM policies results in high maintenance costs and shorter system life. A trade-off between system reliability and maintenance costs should thus be considered in the determination of an optimal PM policy. This study provides a more balanced design of a PM policy for field managers, in which the decrease in the unfixed reliability threshold as the frequency of PM actions increases is considered. Moreover, the proposed model uses the fixed cumulative hazard threshold as a condition variable, and can maintain system reliability above an acceptable level. Hence, it can ensure sustainable system operation and more reflect cost-effectiveness on the model than when the reliability threshold is constrained to be fixed.

In order to demonstrate the efficiency of the proposed model, a numerical example was conducted in two cases, where the cumulative hazard threshold and the predetermined minimum reliability were given as a condition variable, respectively. Moreover, sensitivity analyses were conducted on the condition variables and the maintenance costs to investigate the influential factors in the proposed PM model. The proposed model can decrease the maintenance cost per unit time within the system lifespan by preventing inefficient PM actions. For a sustainable system operation, the field manager may be able to determine a practical PM policy using the proposed model. Additionally, the proposed model can be applied to a continuously monitored system.

\section{ACKNOWLEDGMENT}

This work was supported by the National Research Foundation of Korea(NRF) grant funded by the Korean government(MSIP) (No. NRF2018R1A2B6003232).

\section{REFERENCES}

[1] R. Barlow and L. Hunter, "Optimum preventive maintenance policies," Operations research, vol. 8, no. 1, pp. 90-100, 1960. https://doi.org/10.1287/opre.8.1.90

[2] T. Nakagawa, "Optimum policies when preventive maintenance is imperfect," IEEE Transactions on Reliability, vol. 4, pp. 331-332, 1979. https://doi.org/10.1109/TR.1979.5220624

[3] R. Canfield, "Cost optimization of periodic preventive maintenance," IEEE Transactions on Reliability, vol. 35, no. 1, pp. 78-81, 1986.

https://doi.org/10.1109/TR.1986.4335355
[4] R. H. Yeh and W. L. Chang, "Optimal threshold value of failure-rate for leased products with preventive maintenance actions," Mathematical and Computer Modelling, vol. 46, no. 5, pp. 730-737, 2007.

https://doi.org/10.1016/j.mcm.2006.12.001

[5] Y. Zhao, "On preventive maintenance policy of a critical reliability level for system subject to degradation," Reliability Engineering \& System Safety, vol. 79, no. 3, pp. 301-308, 2003.

https://doi.org/10.1016/S0951-8320(02)00201-6

[6] X. Zhou, L. Xi, and J. Lee, "Reliability-centered predictive maintenance scheduling for a continuously monitored system subject to degradation," Reliability Engineering \& System Safety, vol. 92, no. 4, pp. 530-534, 2007

https://doi.org/10.1016/j.ress.2006.01.006

[7] S. Martorell, A. Sanchez, and V. Serradell, "Age-dependent reliability model considering effects of maintenance and working conditions," Reliability Engineering \& System Safety, vol. 64, no. 1, pp. 19-31, 1999.

https://doi.org/10.1016/S0951-8320(98)00050-7

[8] S. El-Ferik and M. Ben-Daya, "Age-based hybrid model for imperfect preventive maintenance," IIE Transactions, vol. 38, no. 4 , pp. 365-375, 2006.

https://doi.org/10.1080/07408170500232545

[9] D. Lin, M. J. Zuo, and R. C. Yam, "Sequential imperfect preventive maintenance models with two categories of failure modes," Naval Research Logistics, vol. 48, no. 2, pp. 172-183, 2001.

https://doi.org/10.1002/1520-6750(200103)48:2<172::AIDNAV5>3.0.CO;2-5

[10] Z. L. Lin and Y. S. Huang, "Nonperiodic preventive maintenance for repairable systems," Naval Research Logistics (NRL), vol. 57, no. 7, pp. 615-625, 2010. https://doi.org/10.1002/nav.20418

[11] Z.-L. Lin, Y.-S. Huang, and C.-C. Fang, "Non-periodic preventive maintenance with reliability thresholds for complex repairable systems," Reliability Engineering \& System Safety, vol. 136, pp. 145-156, 2015.

https://doi.org/10.1016/j.ress.2014.12.010

\section{AUTHOR PROFILE}

Juhyun Lee is a Ph.D candidate of Industrial and management engineering at the Hanyang University, South Korea. He received his bachelor's degree in Industrial and management engineering in 2013 from Hanyang University. His research interests include Bayesian probability modeling, reliability engineering, and maintenance modeling.

Jihyun Park is a Ph.D candidate of Industrial and management engineering at the Hanyang University, South Korea. He received his bachelor's degree in Industrial and management engineering in 2013 from Hanyang University. His research interests include Bayesian probability modeling, reliability engineering, and risk assessment methodology.

Suneung Ahn is a Professor of Industrial and management engineering at the Hanyang University ERICA, South Korea. He received his Ph.D. degree in Industrial Engineering \& Operations Research in 1995 from University of California at Berkeley. His papers have been published in Applied Mathematical Modeling, Computer and Operation Research, Journal of the Operational Research Society, Stochastic Environmental Research and Risk Assessment, etc. His research interests include Bayesian probability modeling, Reliability Engineering. 